Check for updates

Cite this: RSC Adv., 2019, 9, 18688

Received 5th May 2019

Accepted 30th May 2019

DOI: $10.1039 / c 9 r a 03337 h$

rsc.li/rsc-advances

\title{
PEGylated polyvinylidene fluoride membranes via grafting from a graphene oxide additive for improving permeability and antifouling properties
}

\begin{abstract}
Bin Chen, ${ }^{a}$ Yan Zhang, ${ }^{\text {ab }}$ Jialu Zhang, ${ }^{\text {ab }}$ Lijing Zhu (D)*b and Haichao Zhao (D) *b
Polyvinylidene fluoride (PVDF) porous membranes with enhanced hydrophilicity and antifouling performance were developed via surface PEGylation (PEG, polyethylene glycol) via a reactive graphene oxide (GO) additive. PVDF/GO blended membranes were first fabricated via a non-solvent-induced phase separation process. Then the carboxyl groups of GO sheets immobilized on the membrane surface acted as initiating sites for grafting amine-functionalized PEG (PEG- $\left.\mathrm{NH}_{2}\right)$ chains via an amination reaction. Analysis of the X-ray photoelectron spectroscopy and Fourier transform infrared spectroscopyattenuated total reflectance results confirmed the successful grafting of hydrophilic PEG molecular chains on PVDF membrane surfaces. The water contact angle of the PEGylated PVDF membrane decreased to $59.9^{\circ}$, indicating improved hydrophilicity. As a result, the antifouling performance was enhanced significantly. After surface PEGylation, the flux recovery rate is reached $90.2 \%$, the total fouling ratio was as low as $20.7 \%$, and reversible fouling plays a dominant role during the membrane fouling process. This work provides a valuable strategy to fabricate PEGylated membranes via the introduction of a reactive $\mathrm{GO}$ additive.
\end{abstract}

\section{Introduction}

On Earth, water is an important natural resource. Seventy-two percent of the Earth's surface is covered by water, but fresh water resources account for only $0.5 \%$ of the total. ${ }^{1}$ With the development of the world economy, the population continues to grow, cities continue to increase and expand, and the increase in water consumption leads to depletion of the Earth's resources, lack of water resources and serious damage to water resources. Fresh water shortages in ecological environments are mainly caused by domestic sewage and industrial wastewater. ${ }^{2,3}$ Therefore, how to obtain fresh water is a huge challenge for us. At present, membrane separation is one of the best technologies for water purification owing to its ease of operation, low energy consumption and low cost. $^{4-6}$ Porous membranes, such as microfiltration membranes, ion-water separation membranes, reverse osmosis membranes, ultrafiltration membranes and gas membranes, allow the passage of one or more substances, but at the same time are impermeable to other materials, such as

${ }^{a}$ School of Materials Science and Engineering, Shenyang University of Chemical Technology, 11 St. Economic \& Technological Development Zone, Shenyang 110142, P. R. China

${ }^{b}$ Key Laboratory of Marine Materials and Related Technologies, Zhejiang Key Laboratory of Marine Materials and Protective Technologies, Ningbo Institute of Materials Technology and Engineering, Chinese Academy of Science, Ningbo 315201, P. R. China.E-mail: zhulijing@nimte.ac.cn; zhaohaichao@nimte.ac.cn contaminants, in order to achieve separation and purification. $^{7-10}$

Polyvinylidene fluoride (PVDF) is a promising polymer material owing to its excellent properties, such as high mechanical strength, unique antioxidant activity, excellent chemical resistance, good thermal stability and good membrane-forming ability. ${ }^{\mathbf{1 1 - 1 5}}$ However, the strong hydrophobicity and low surface energy of PVDF membrane make it easily contaminated by proteins and bacteria when treating wastewater, resulting in a decrease in water flux and a short lifespan. ${ }^{\mathbf{1 3 , 1 6 - 1 8}}$ Therefore, it is necessary to improve the antifouling performance of PVDF membrane. At present, many methods have been proposed: PVDF membrane can be optimized by copolymerization, grafting, coating, ion treatment, mixing and other methods. ${ }^{19-22}$ As a high-performing and promising additive, polyethylene glycol (PEG) or PEG-containing material was added to membranes, improving their hydrophilicity and antifouling performance. ${ }^{23,24}$ PEG is a non-ionic water-soluble polymer, chemically stable in air and solution, unsuitable for microbial growth, and not easy to spoil. Its highly hydrophilic group can form an aqueous layer on the membrane surface to enhance the hydrophilic performance and antifouling performance of the membranes. ${ }^{25-27}$

Currently, two-dimensional nanomaterials (graphene oxide (GO), boron nitride (BN), molybdenum disulfide $\left(\mathrm{MoS}_{2}\right)$, etc.) also have been applied to improve the antifouling of the membranes. ${ }^{28-31} \mathrm{GO}$, an important derivative of graphene, includes various oxygen-containing functional groups (for 
example, hydroxyl group, epoxy group, carbonyl group, etc.). ${ }^{32,33}$ It has been widely used to modify PVDF membranes. GO sheets have been added directly into the casting solution to prepare blended membranes with high flux due to the laminated structure of GO sheets. Conductive PVDF-GO-Ni membrane was also obtained that achieved highly efficient performance. ${ }^{34}$ In addition, GO sheets were coated on membrane surfaces to improve their hydrophilicity and antifouling performance..$^{27,35,36}$

In this study, PVDF/GO blended membranes were first fabricated by non-solvent-induced phase separation (NIPS). Then PEG molecular chains were grafted onto the reactive GO sheets via amination. The surface chemical composition, hydrophilicity, permeability and antifouling performance of the PEGylated PVDF/GO membranes were explored. The results in this work proved that the GO sheets additive can be employed to provide active sites to further modify the porous membranes, and this will be of great significance for subsequent studies.

\section{Experimental}

\section{Materials}

PVDF (FR-1015) was obtained from Solvay (Shanghai Agency, P. R. China). Graphene oxide was purchased from Yuhuang Technology Co., Ltd. Bovine serum albumin (BSA, $M_{\mathrm{w}}=66.4 \times$ $10^{3} \mathrm{~g} \mathrm{~mol}^{-1}$ ) was obtain from Aladdin Reagent Co., China. Methoxypolyethylene glycols (MPEG, $M_{\mathrm{w}}=750 \mathrm{Da}$, 99\%), 2-(tertbutoxycarbonylamino)-1-ethanol, dioxane (99\%), hydrochloric acid (99\%), ethyl acetate (99\%), trifluoroacetic acid, sodium carbonate (99\%), toluene-2,4-diisocyanate (TDI, 99\%) and $N, N$ dimethylacetamide (DMAc, 99\%) were purchased from Sinopharm Chemical Reagent. All chemicals were reagent grade.

\section{Synthesis of PEG-NH}

Di-tert-butyloxycarbonate $\left((\mathrm{BOC})_{2} \mathrm{O}, 100 \mathrm{mmol}\right)$ was dissolved in a suitable amount of 1,4-dioxane as solvent and the solution was added slowly into $100 \mathrm{mmol}$ mono-2-(tertbutoxycarbonylamino)-1-ethanol. The mixture was stirred for one day, then extracted with ethyl acetate and evaporated to provide amino-protected 2-(tert-butoxycarbonylamino)-1ethanol under ice bath conditions. MPEG (25 mmol) was vacuum dried at $80{ }^{\circ} \mathrm{C}$ for $6 \mathrm{~h}$ and added into toluene-2,4diisocyanate (TDI, $25 \mathrm{mmol}$ ) at $25{ }^{\circ} \mathrm{C}$. The mixture was kept at $55{ }^{\circ} \mathrm{C}$ with stirring for $3 \mathrm{~h}$, and $25 \mathrm{mmol}$ of amino-protected 2(tert-butoxycarbonylamino)-1-ethanol was added under stirring for $3 \mathrm{~h}$. Trifluoroacetic acid (TFA, $50 \mathrm{mmol}$ ) was added to remove the amino-protecting group in order to obtain PEG- $\mathrm{NH}_{2}$ by mechanical stirring for $6 \mathrm{~h}$ and evaporating to remove excess solvent. Anhydrous sodium carbonate $\left(\mathrm{K}_{2} \mathrm{CO}_{3}\right)$ and ethyl acetate were added dropwise into the solution until the $\mathrm{pH}$ was over 7. A clear yellow thick liquid ( $\mathrm{PEG}-\mathrm{NH}_{2}, 24.3 \mathrm{~g}, M_{\mathrm{w}}=985.23 \mathrm{Da}$ ) was obtained after drying in vacuum. ${ }^{37,38}$

\section{Fabrication of membranes}

PEGylated PVDF/GO membranes were fabricated via a surface grafting method as shown in Scheme 1. PVDF/GO blended membranes were prepared by non-solvent induced phase separation (NIPS).$^{39}$ PVDF powder was dried in a vacuum oven at $80{ }^{\circ} \mathrm{C}$ for $6 \mathrm{~h}$ to remove the absorbed water. GO was dispersed in DMAc by sonication for $120 \mathrm{~min}$ to prepare a homogeneous solution. Then, $15 \mathrm{wt} \%$ PVDF was added to the solution under stirring at $60{ }^{\circ} \mathrm{C}$ for $10 \mathrm{~h}$ to obtain a casting solution. The solution was evacuated for $5 \mathrm{~h}$ and left at room temperature for one day to remove air bubbles. The solution was cast on a glass plate using a $250 \mu \mathrm{m}$ casting knife, and immediately immersed in a water coagulation bath at room temperature. The fabricated PVDF/GO blended membranes were respectively named M0, M0.5, M1 and M2 according to the weight concentrations of GO in the PVDF powder. PEGylated PVDF/GO membranes were fabricated by the surface grafting technique. In a typical process, M1 was soaked in PEG- $\mathrm{NH}_{2}$ solution $\left(2 \mathrm{~g} \mathrm{~L}^{-1}\right)$ at $90{ }^{\circ} \mathrm{C}$ for $24 \mathrm{~h}$. Then, washed with deionized water, the obtained membrane was dried and named M1- $g$-PEG.

\section{Characterization}

Raman spectroscopy of GO was conducted using a confocal Raman spectrometer (inVia Reflex, Renishaw, UK) at a wavelength of $532.8 \mathrm{~nm}$. X-ray photoelectron spectroscopy (XPS; Kratos Analytical, UK) was used to study the surface chemical compositions of the membranes. UV-vis spectra were recorded using a Lambda 950 UV-vis spectrometer (PerkinElmer, USA). The functional groups and chemical structures of the membranes were characterized by Fourier transform infrared spectroscopy-attenuated total reflectance (FTIR-ATR; Cary $660+620$, USA) at room temperature. The surface and crosssectional morphologies of the membranes were examined by thermal field emission scanning electron microscopy (TFESEM; Verios G4 UC, Thermo Scientific, USA). To prepare the SEM samples, the membranes were cut into small pieces and immersed in liquid nitrogen for 60-90 s. The frozen membranes were snapped with a pair of tweezers. After drying at room temperature, the samples were adhered to the TFSEM sample holder with conductive tape and a thin Pt layer was sputtered on. The roughness of each membrane surface was observed by atomic force microscopy (AFM; Dimension 3100, Vecco, USA) with an area of $3 \mu \mathrm{m} \times 3 \mu \mathrm{m}$.

\section{Water contact angle (WCA) measurements}

WCA was measured using a contact angle measuring device (OCA 20, Dataphysics, Germany) based on the sessile drop method. The sample was dried at room temperature for $6 \mathrm{~h}$. Deionized water $(2 \mu \mathrm{L})$ was dripped onto the surface of the flat membrane using a micro syringe. The initial water contact angle on the surface was measured. The contact angles were measured at least 7 times at random positions on each membrane to obtain a reliable average value.

\section{Water flux and antifouling performance}

The antifouling experiment was divided into three parts. The membrane was cut into a circle of suitable size and then fixed on the sample holder of a dead-end stirred filtration cell (Millipore Corporation, XFUF04701, USA) with an effective area of $14.2 \mathrm{~cm}^{2}$. At room temperature, the membrane was 


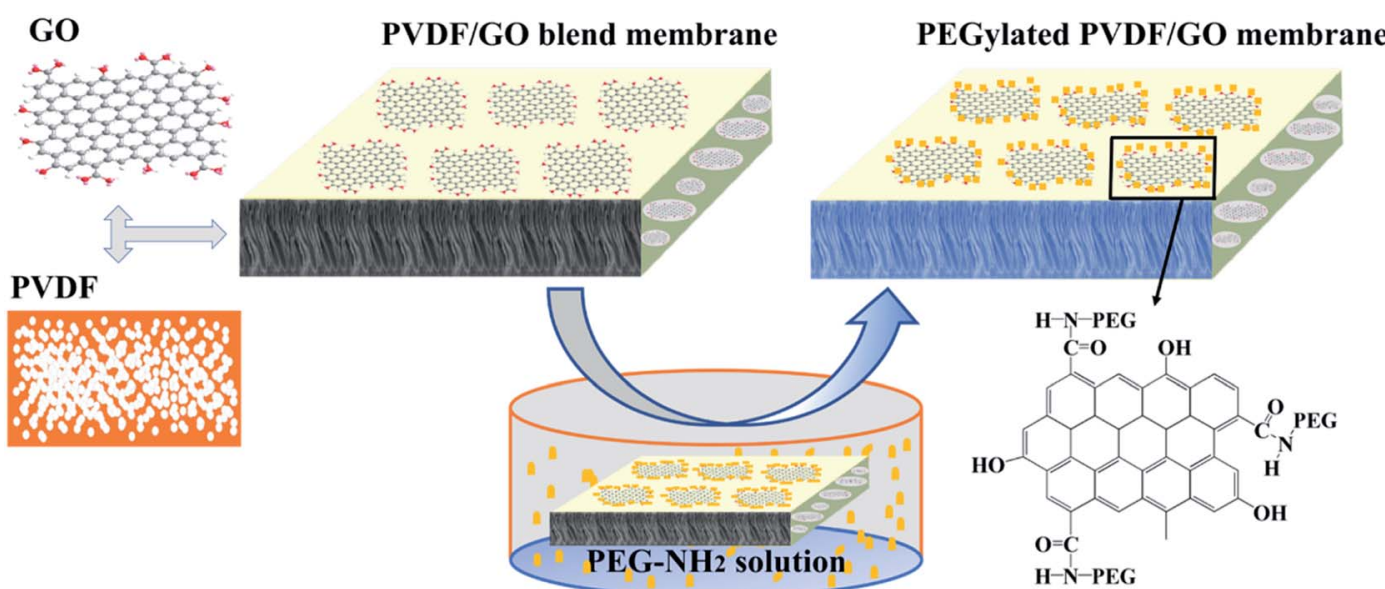

Scheme 1 A schematic illustration of the fabrication of the PEGylated PVDF/GO membranes.

Table 1 Surface compositions of M0, M1 and M1-g-PEG

\begin{tabular}{lllll}
\hline Sample & C1s (mol\%) & F1s (mol\%) & O1s (mol\%) & N1s (mol\%) \\
\hline M0 & 48.3 & 51.5 & 0.2 & - \\
M1 & 57.6 & 31.5 & 9.1 & 1.8 \\
M1-g-PEG & 51.5 & 30.6 & 14.8 & 3.1
\end{tabular}

first compacted at a pressure of 2 bar for $1 \mathrm{~h}$ to obtain a stable flow. The pressure was reduced to 1 bar, and the deionized water flow rate was recorded every minute. The corresponding water flux was calculated according to eqn (1) and named $J_{1}$. Then, $0.8 \mathrm{~g} \mathrm{~L}^{-1}$ BSA solution in physiological saline was permeated through the membrane sample at a stirring rate of $500 \mathrm{rpm}$ to minimize concentration polarization. After filtering for $30 \mathrm{~min}$ at 1 bar, BSA flux $\left(J_{2}\right)$ was calculated. After BSA solution filtration, the membrane was washed with physiological saline under shaking for $24 \mathrm{~h}$. Finally, the deionized water was again pushed through the cleaned membrane, and water flux was measured again and recorded as $J_{3}$.
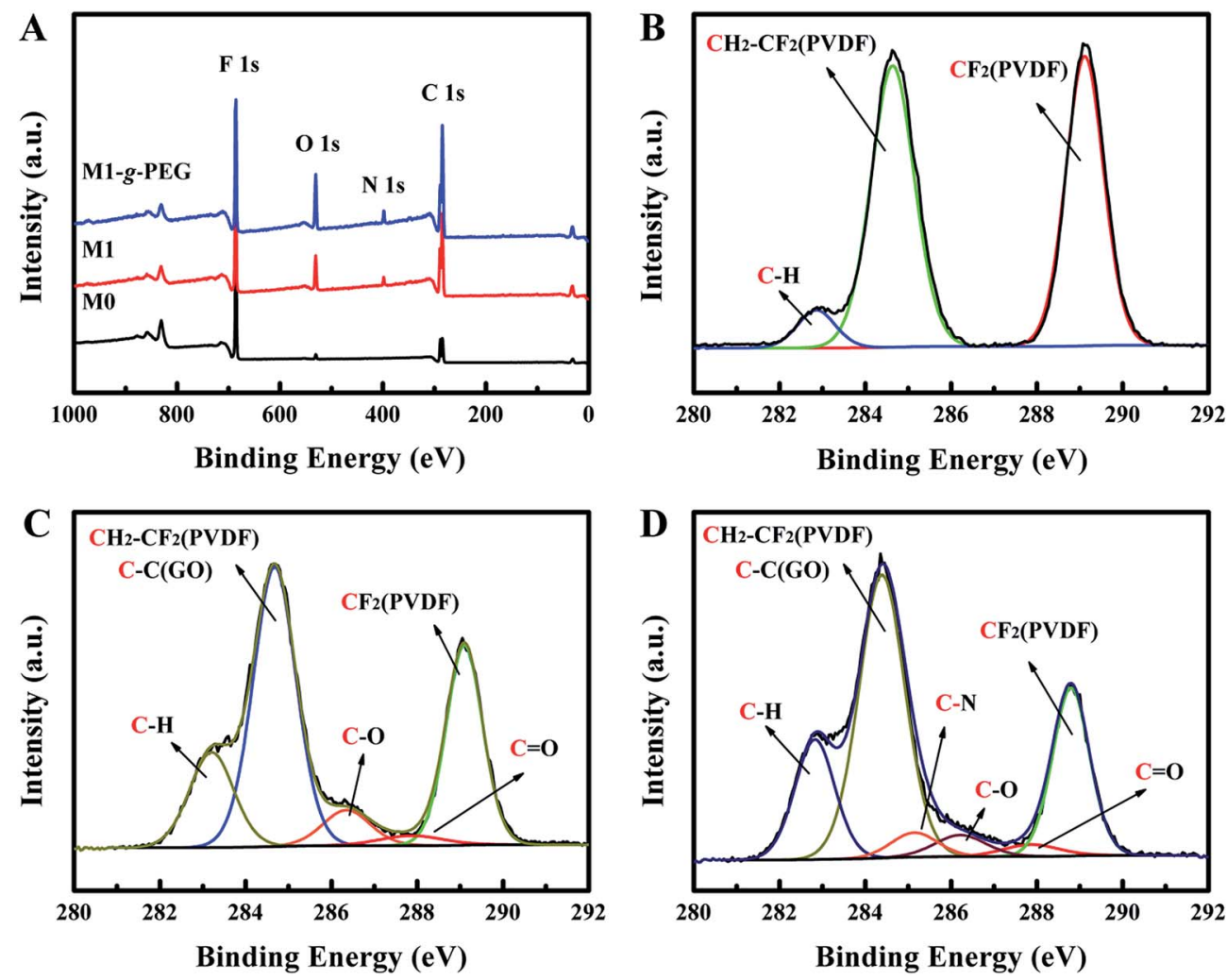

Fig. 1 XPS wide scans (A) and C1s core-level spectra of M0 (B), M1 (C), and M1-g-PEG (D). 
$\mathbf{A}$

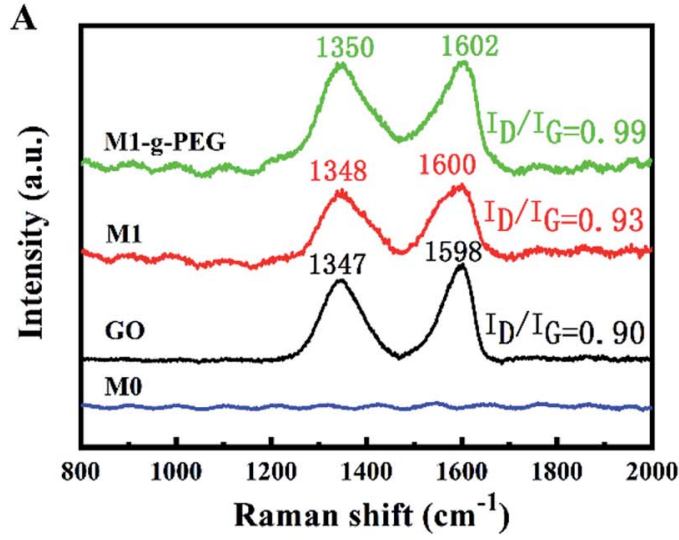

C
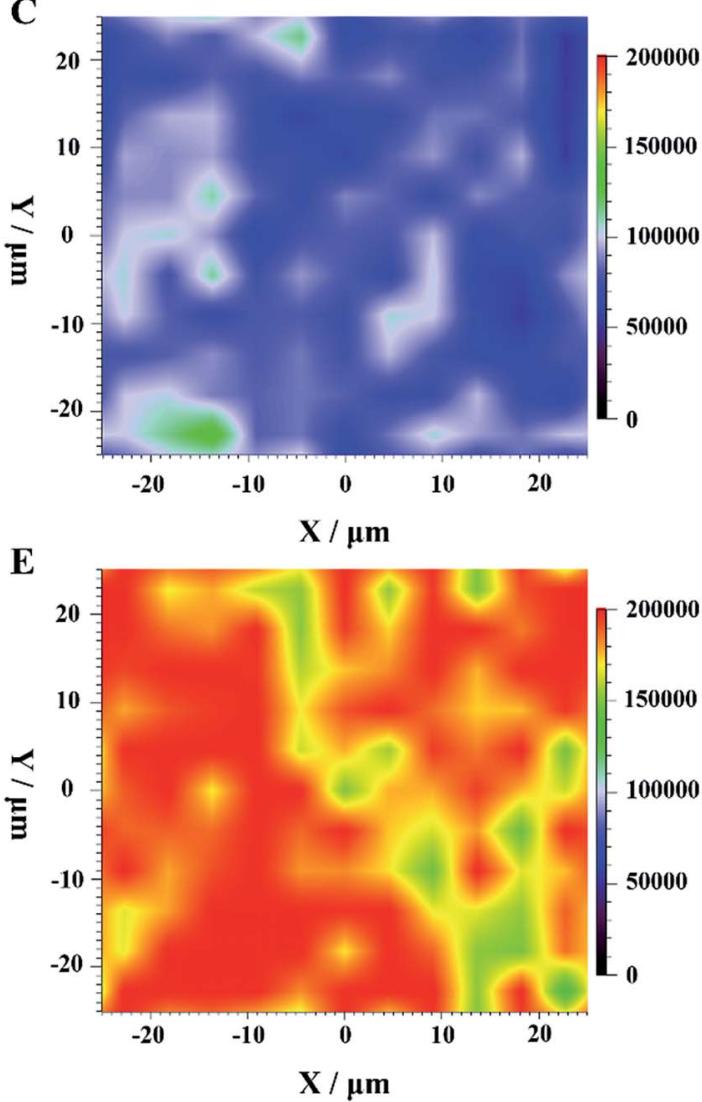

B

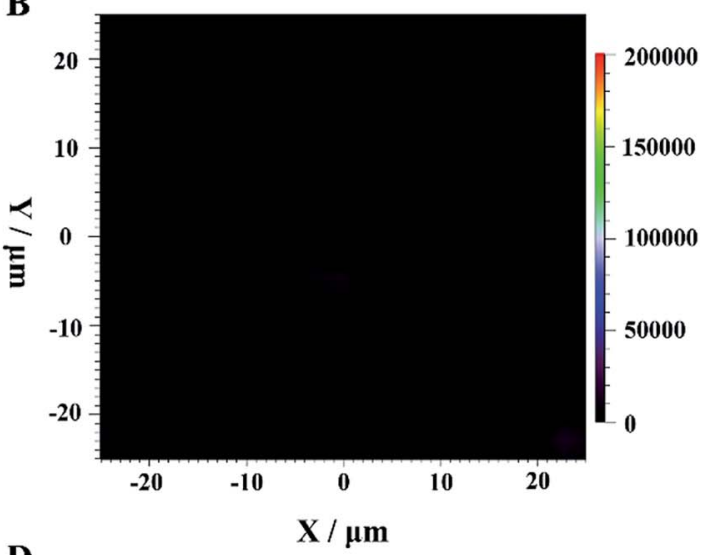

D
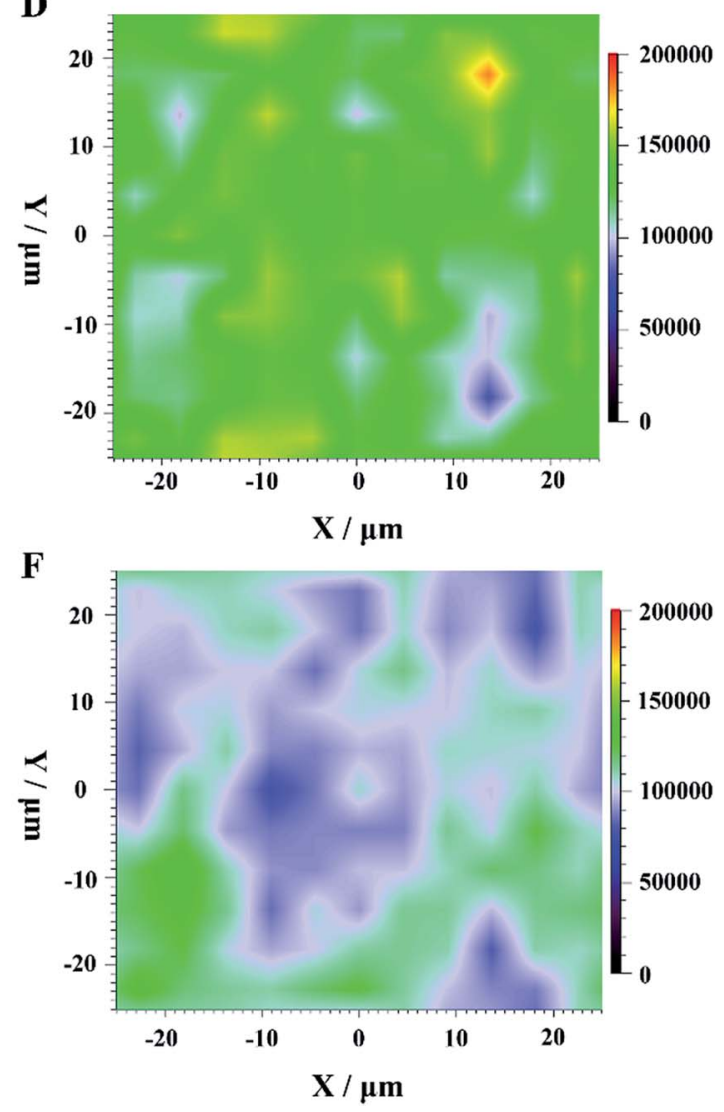

Fig. 2 Raman spot-scanning (A) and map-scanning images of M0 (B), M0.5 (C), M1 (D), M2 (E) and M1-g-PEG (F).

$$
J=\frac{V}{S \times t} \times 100 \%
$$

where $J\left(\mathrm{~L} \mathrm{~m}^{-2} \mathrm{~h}^{-1}\right)$ is the flux, $V(\mathrm{~L})$ is the volume of the collected liquid, $S\left(14.2 \mathrm{~cm}^{2}\right)$ is the effective area, and $t$ is the running time of the liquid.

The protein rejection $(R)$ was calculated using the equation:

$$
R=\left(1-\frac{C_{1}}{C_{2}}\right) \times 100 \%
$$

where $C_{1}$ and $C_{2}$ are the BSA concentrations of permeate and feed solutions, respectively. They were measured with a UV-vis spectrophotometer (Lambda 950, PerkinElmer, USA) at $280 \mathrm{~nm}$.

The antifouling performance of the membrane was evaluated by flux recovery rate (FRR), total fouling ratio $\left(R_{\mathrm{t}}\right)$, reversible fouling rate $\left(R_{\mathrm{r}}\right)$ and irreversible fouling rate $\left(R_{\mathrm{ir}}\right)$.

$$
\begin{gathered}
\mathrm{FRR}=\frac{J_{3}}{J_{1}} \times 100 \% \\
R_{\mathrm{t}}=\frac{J_{1}-J_{2}}{J_{1}} \times 100 \%
\end{gathered}
$$



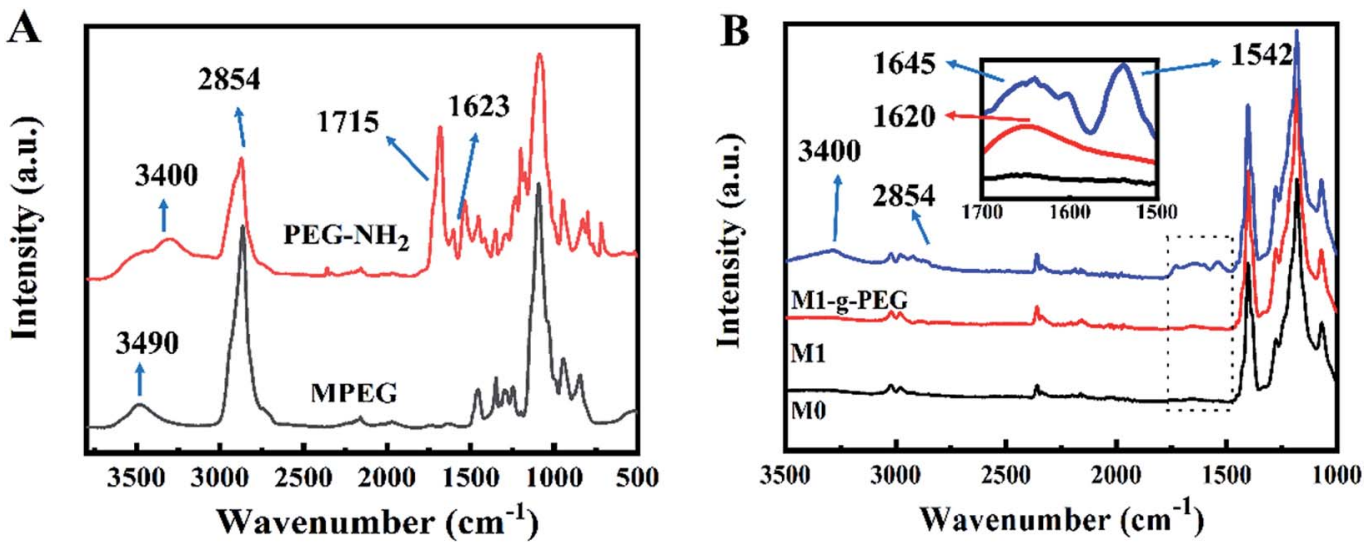

Fig. 3 FTIR-ATR spectra of MPEG and PEG-NH $2(A)$ and the membranes of MO, M1 and M1-g-PEG (B).
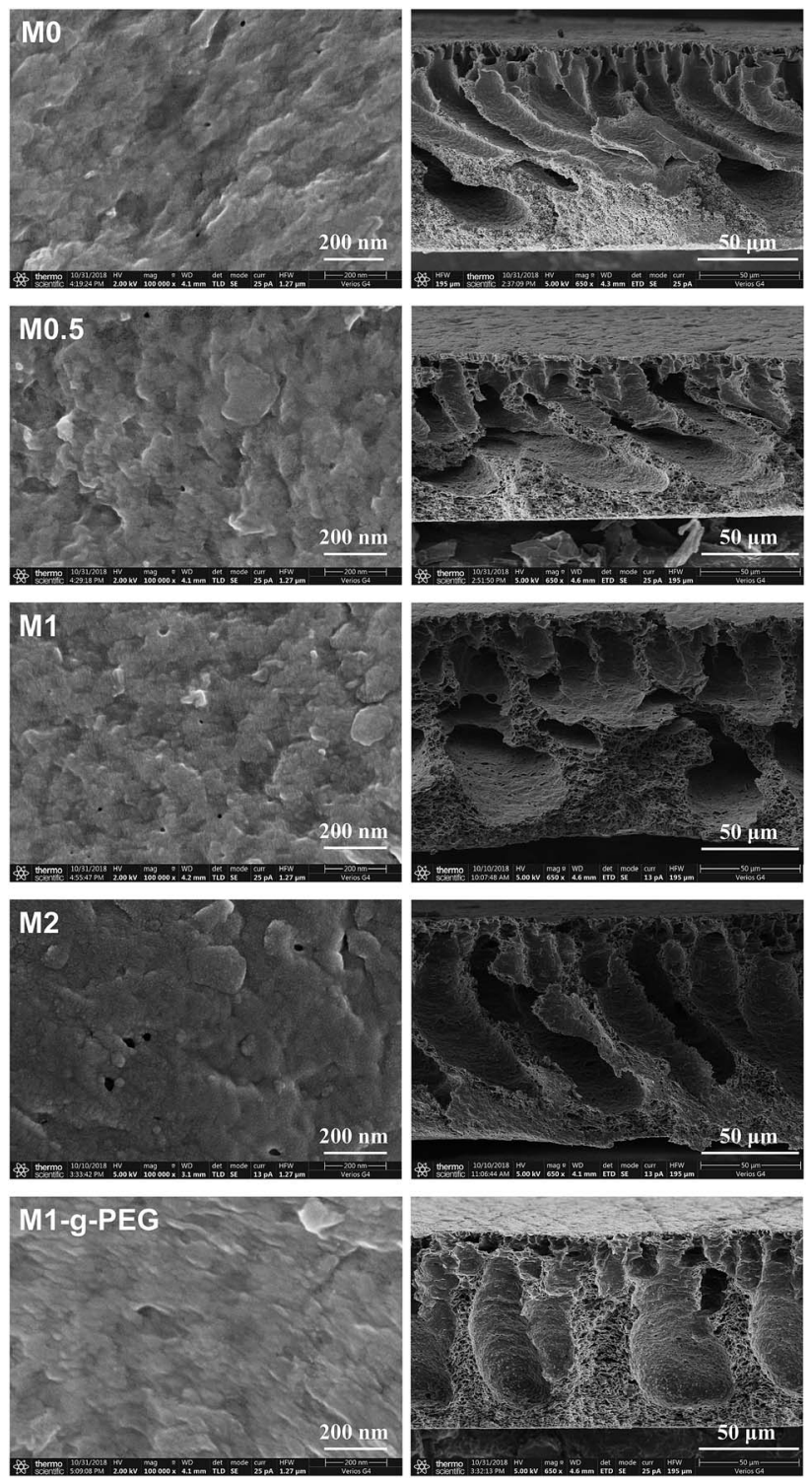

Fig. 4 Open side surface and cross-section SEM images of the membranes.

$$
\begin{gathered}
R_{\mathrm{r}}=\frac{J_{3}-J_{2}}{J_{1}} \times 100 \% \\
R_{\text {ir }}=\frac{J_{1}-J_{3}}{J_{1}} \times 100 \%
\end{gathered}
$$

\section{Results and discussion}

\section{Surface compositions of the membranes}

XPS measurement was used to identify the chemical compositions of the membrane surfaces, and typical results and spectra are presented in Table 1 and Fig. 1, respectively. It can be observed that the wide scan of M1 contains the elements C, N, O and F. After introducing GO, the content of the element $\mathrm{F}$ declines from $51.5 \%$ (M0) to $31.5 \%$ (M1), while the O percentage rises from $0.2 \%$ (M0) to $9.1 \%$ (M1). This is caused by the high percentage of $\mathrm{C}$ and $\mathrm{O}$ in GO. It should be noticed that $\mathrm{N}$ of M1 $(1.8 \%)$ may originate from the original GO material. For M1- $g$ $\mathrm{PEG}$, the $\mathrm{O}$ concentration increases to $14.8 \%$. To provide more information concerning the chemical changes of the membrane surfaces, the $\mathrm{C} 1 \mathrm{~s}$ core-level spectrum of $\mathrm{M} 0$ was resolved into peaks including $\mathrm{C}-\mathrm{H}, \mathrm{CH}_{2}-\mathrm{CF}_{2}$ and $\mathrm{CF}_{2}$ (Fig. 1B). In Fig. 1C, the $\mathrm{C} 1 \mathrm{~s}$ core-level spectrum of $\mathrm{M} 1$ was resolved into five peaks, derived from $\mathrm{C}-\mathrm{H}, \mathrm{CH}_{2}-\mathrm{CF}_{2}$ plus $\mathrm{C}-\mathrm{C}, \mathrm{C}-\mathrm{O}, \mathrm{C}=\mathrm{O}$ and $\mathrm{CF}_{2}$. The peaks for $\mathrm{CH}_{2}-\mathrm{CF}_{2}$ and $\mathrm{C}-\mathrm{C}$ coincide, and the $\mathrm{C}-\mathrm{O}$ and $\mathrm{C}=\mathrm{O}$ peaks belong to the GO sheets. For M1-g-PEG, a new C-N peak at $285.7 \mathrm{eV}$ appears (Fig. 1D). All results indicate that PEGylated PVDF membranes have been successfully fabricated with the aid of the reactive GO sheets additive.

Raman spectroscopy is an effective method for characterizing the structure of carbon materials. As shown in Fig. 2A, M0 has no apparent Raman signal in the range of $800-2000 \mathrm{~cm}^{-1}$. In contrast, $\mathrm{GO}$ exhibits a D band at $1347 \mathrm{~cm}^{-1}$ and a $\mathrm{G}$ band at $1558 \mathrm{~cm}^{-1}$. The broad D band suggests that some carbon atoms of GO have been converted into $\mathrm{sp}^{3}$ hybrid structure. The D band variation may be a structural defect caused by epoxides and hydroxyl groups on the carbon-based surface, and the $\mathrm{G}$ band is caused by the in-plane displacement of carbon atoms in the hexagonal carbon sheet. In the Raman spectra of M1 and 

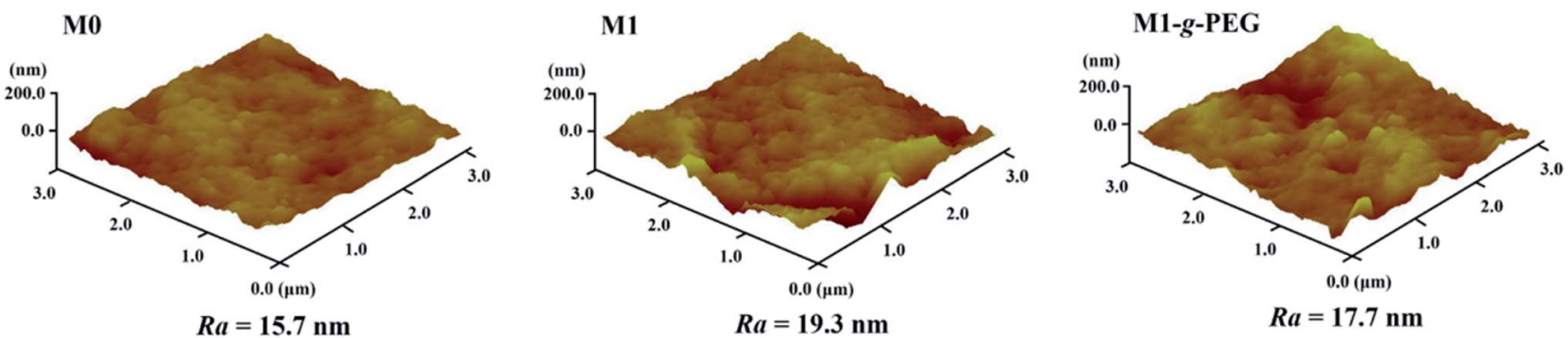

Fig. 5 AFM images of MO, M1 and M1-g-PEG.

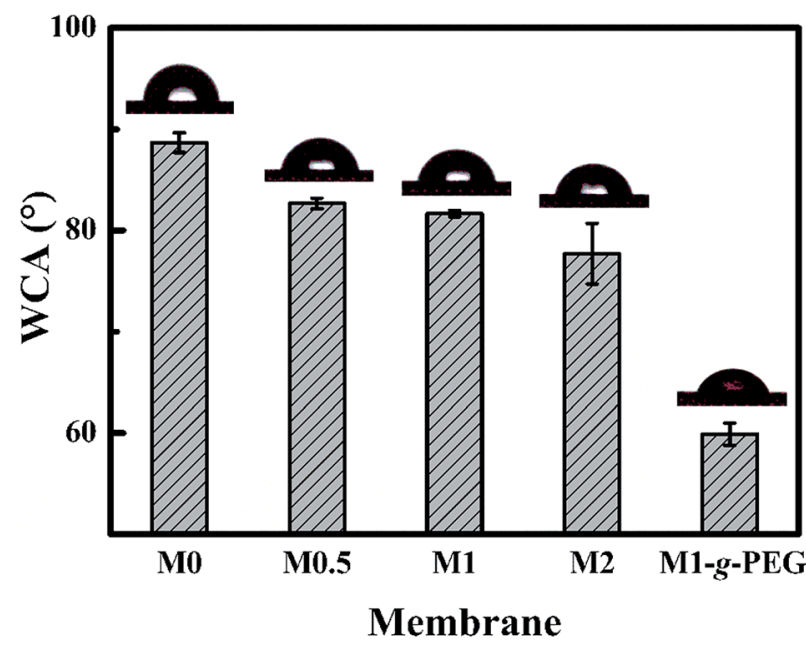

Fig. 6 The water contact angles of M0, M0.5, M1, M2 and M1-g-PEG.

M1- $g$-PEG, D and G bands are observed. Generally, the intensity ratio of the $\mathrm{D}$ band to the $\mathrm{G}$ band $\left(I_{\mathrm{D}} / I_{\mathrm{G}}\right)$ is used to evaluate the degree of defects in the carbon materials. The ratio of $I_{\mathrm{D}} / I_{\mathrm{G}}$ increases from 0.90 (M0) to 0.93 (M1) and 0.99 (M1-g-PEG), indicating that the functional groups of GO on the surfaces of the membrane were reacted or eliminated. So, this indicates that the epoxy group and carboxyl group of GO may react with PEG-NH $\mathrm{N}_{2}$. Raman map-scanning test images of the membranes at $50 \times 50 \mu \mathrm{m}$ under the $\mathrm{G}$ peak range of GO are shown in Fig. 2B-F. In contrast to M0, the PVDF/GO blended membranes produce colored images, indicating GO sheets have been immobilized on the membranes. And the color changes from blue (M0.5) to green (M1) and red (M2), indicating high GO concentration on the surfaces. In addition, the color of the images remains consistent over a large area, indicating that GO is distributed homogeneously on the surfaces. In Fig. 2F, the surface sweeping color of M1- $g$-PEG is lighter than that of M1 (Fig. 2D), indicating less GO on the membrane surface after grafting the PEG- $\mathrm{NH}_{2}$ molecular chains.

FTIR-ATR spectra of MPEG and PEG-NH $\mathrm{N}_{2}$ are shown in Fig. 3A. In the spectrum of MPEG, O-H at $3400 \mathrm{~cm}^{-1}$ and the skeleton peak C-H at $2854 \mathrm{~cm}^{-1}$ are observed. For PEG- $\mathrm{NH}_{2}$, new peaks due to $\mathrm{N}-\mathrm{H}$ at $3400 \mathrm{~cm}^{-1}, \mathrm{C}-\mathrm{H}$ at $2854 \mathrm{~cm}^{-1}, \mathrm{C}=\mathrm{O}$ at $1715 \mathrm{~cm}^{-1}$ and aromatic $\mathrm{C}=\mathrm{C}$ at $1623 \mathrm{~cm}^{-1}$ are present. This indicates that MPEG was aminated successfully. In addition,
FTIR-ATR spectra of the fabricated membranes (M0, M1 and M1- $g$-PEG) are shown in Fig. 3B. In contrast to M0, M1 exhibits the absorption peak of the graphene skeleton at $1620 \mathrm{~cm}^{-1}$. For M1- $g$-PEG, the characteristic peaks at 3400 and $2854 \mathrm{~cm}^{-1}$ derived from PEG are detected, and new peaks at 1645 and $1542 \mathrm{~cm}^{-1}$ are attributed to the amide bonds. The results indicate that PEG chains have been grafted onto the surface of PVDF/GO blended membranes.

\section{Membrane morphologies}

Fig. 4 displays the surface and cross-section SEM images of M0, M0.5, M1, M2 and M1-g-PEG. From the surface images, with the increase of GO content, the pores of the fabricated PVDF/GO blended membranes enlarge. After grafting PEG molecular chains on the surface of M1, the membrane becomes dense. From the cross-sectional images, a typical asymmetric porous structure is divided into two parts, "finger" holes extending from the surface and a dense "sponge" substrate. The asymmetric structure is due to the polymer concentration gradient of the liquid film in a water bath. When the casting solution is immersed, the outer surface of the membranes is immediately solidified, resulting in the formation of dense skin. The solvent present in the casting solution also rapidly diffuses outward, and the diffusion of water causes the membranes to coagulate to create long "finger" holes extending from the surface. As the phase inversion continues, this delamination process between the solvent and water gradually slows down owing to the solidification of the liquid film. As a result, the phase inversion process is inhibited, leading to dense "sponge" pores. ${ }^{40}$

Fig. 5 shows the three-dimensional AFM images of M0, M1 and M1- $g$-PEG. The roughness parameter $R_{\mathrm{a}}$ of the membranes was calculated based on the AFM scan area of $3 \mu \mathrm{m} \times 3 \mu \mathrm{m}$. Compared with M0, more "peaks" are observed on M1 $\left(R_{\mathrm{a}}=\right.$ $19.3 \mathrm{~nm}$ ), indicating a rougher surface. In contrast, the roughness of M1- $g$-PEG is reduced to $17.7 \mathrm{~nm}$ owing to the PEG chains grafted on the membrane surface. After grafting onto the surface of M1, PEG- $\mathrm{NH}_{2}$ molecular segments covered or lapped on the surface, resulting in the reduction of the roughness of the membrane.

\section{Hydrophilicity of membranes}

Water contact angle is used to characterize the hydrophilicity of the membranes. Hydrophilicity is one of the most important 

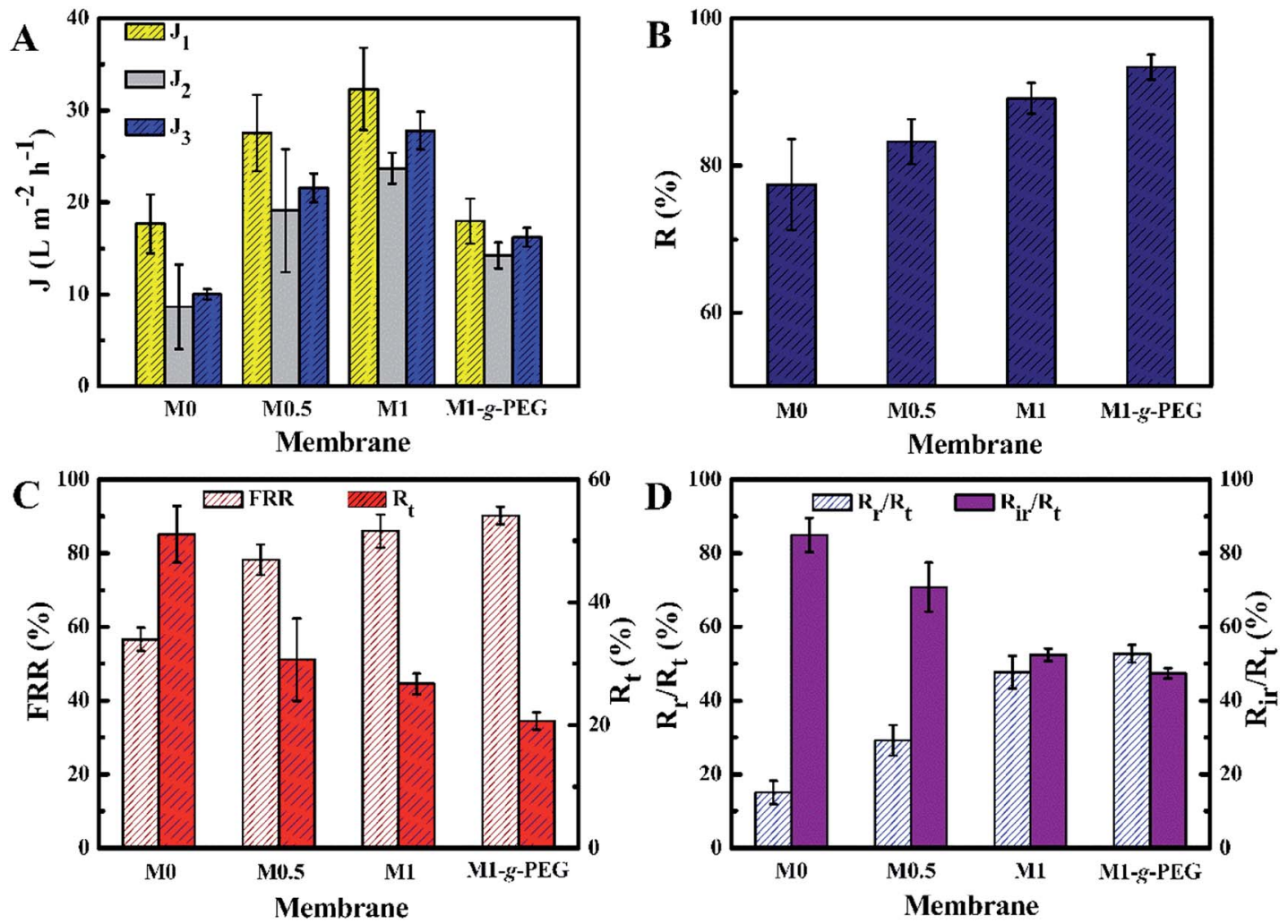

Fig. 7 (A) Pure water flux $\left(J_{1}\right)$, BSA solution flux $\left(J_{2}\right)$ and recovered pure water flux $\left(J_{3}\right)$; (B) water flux recovery rate (FRR) and total fouling rate $\left(R_{\mathrm{t}}\right)$; (C) protein rejection $(R)$; and (D) the ratio of the reversible fouling rate $\left(R_{\mathrm{r}}\right)$ and irreversible fouling rate $\left(R_{\mathrm{ir}}\right)$ to the total fouling rate $\left(R_{\mathrm{r}} / R_{\mathrm{t}}\right.$ and $\left.R_{\mathrm{ir}} / R_{\mathrm{t}}\right)$.

Table 2 Comparative results of water flux recovery, total fouling rate and water contact angle of the membranes

\begin{tabular}{lllll}
\hline Sample & FRR $(\%)$ & $R_{\mathrm{t}}(\%)$ & $\begin{array}{l}\text { Water contact } \\
\text { angle }\left(^{\circ}\right)\end{array}$ & Reference \\
\hline $\mathrm{PVDF} / \mathrm{GO}$ & 85.1 & - & 66.5 & 27 \\
$\mathrm{PVDF} / \mathrm{Al}_{2} \mathrm{O}_{3}$ & 70.0 & - & 70.2 & 45 \\
$\mathrm{PVDF} / \mathrm{GO}$ & 88.56 & - & 60.5 & 46 \\
$\mathrm{PVDF} / \mathrm{GO}$ & 75.4 & 24.0 & - & 47 \\
$\mathrm{M} 1-\mathrm{g}$-PEG & 90.2 & 20.7 & 60.1 & This work \\
& & & &
\end{tabular}

properties of membranes because it affects their flux and antifouling performance. As shown in Fig. 6, the contact angle of M1 was as high as $90^{\circ}$, indicating poor hydrophilicity. The hydrophilicity increased after the hydrophilic GO sheets were added, and the water content angle of M1-g-PEG achieved 59.9 ${ }^{\circ}$. A hydrophilic layer will be formed between the hydrophilic membrane surface and water for further promoting liquid permeability and antifouling performance.

\section{Water flux and antifouling performance of the membranes}

Pure water flux $\left(J_{1}\right)$ and BSA rejection $(R)$ of the fabricated membranes were characterized by using a dead-end stirred filter at 1 bar pressure, and the results are shown in Fig. 7A and B. As seen, the water flux of PVDF/GO blended membranes is higher than that of M0. ${ }^{41} J_{1}$ increases from 17.7 to $32.5 \mathrm{~L} \mathrm{~m}^{-2} \mathrm{~h}^{-1}$ when the GO sheets content increases from 0 to $2 \mathrm{wt} \%$. $J_{1}$ of M1- $g$-PEG is lower than that of other membranes, while $R$ is as high as $93.4 \%$, owing to its dense surface (Fig. 4). Moreover, BSA was used as the model foulant to characterize the antifouling performances of the membranes before and after surface modification. ${ }^{27,42,43}$ As shown in Fig. 7C, for the fabricated PVDF/GO membranes, FRR increases from 56.6 to $86.0 \%$ and $R_{\mathrm{t}}$ decreases from 51.1 to $26.7 \%$ when the content of GO rises from 0 to $1 \mathrm{wt} \%$. After surface PEGylation, FRR of M1- $g$-PEG achieves $90.2 \%$, while $R_{\mathrm{t}}$ is as low as $20.7 \%$. The smaller $R_{\mathrm{t}}$ indicates less protein adsorbs to or deposits on the membrane surfaces. In addition, the ratio of reversible fouling rate to the total fouling rate $\left(R_{\mathrm{r}} / R_{\mathrm{t}}\right)$ of M1- $g$-PEG is higher than that of other membranes (Fig. 7D). M1-g-PEG has not only lower $R_{\mathrm{t}}$ but also lower $R_{\text {ir }}$ during BSA filtration, and the reversible fouling is the dominant factor responsible for the flux loss, the smaller $R_{\mathrm{t}}$ and $R_{\mathrm{ir}}$ values, and the better antifouling performance of the membranes. The improvement in the antifouling performance of PVDF membranes is mainly attributed to the enhanced hydrophilicity. Hydrophilic groups of PEG and the remaining hydroxyl/carboxyl groups of GO may interact with water molecules through van der Waals forces and hydrogen bonds forming a water molecular layer on M1- $g$-PEG surface to prevent or reduce BSA adhesion/ deposition, resulting in high antifouling performance..$^{27,44}$ Compared with the membrane data in some literature (Table 2), the antifouling performance and hydrophilicity of M1- $g$-PEG are better. And the reversible fouling plays a dominant role during the membrane fouling process. 


\section{Conclusions}

In this work, a PVDF/GO blended membrane and its PEGylated membrane formed by grafting PEG onto the graphene oxide additive were prepared via NIPS with the aim of improving the permeability and antifouling performance. The addition of GO sheets significantly promoted the water flux and hydrophilicity of PVDF membranes, and improved the antifouling performance. Furthermore, the functional groups of GO sheets can act as reactive sites for the immobilization of hydrophilic PEG chains. After surface PEGylation, the hydrophilicity of M1- $g$-PEG was further enhanced; the water contact angle was reduced to $59.9^{\circ}$. Moreover, FRR of M1- $g$-PEG increased to $90.2 \%$, while $R_{\mathrm{t}}$ decreased to $20.7 \%$ compared with non-modified PVDF membrane. The results indicated that the fabricated PEGylated PVDF/GO membranes exhibited enhanced hydrophilicity and antifouling performance. This work provides a valuable strategy to fabricate PEGylated membranes via the introduction of a reactive $\mathrm{GO}$ additive into the membrane matrix.

\section{Conflicts of interest}

The authors declare no potential conflicts of interest with respect to the research, authorship, and/or publication of this article.

\section{Acknowledgements}

This study was supported by the National Science Foundation of China (No. 51603214), the National Science and Technology Bureau (No. 2018A610110), and the "One hundred Talented People” of Chinese Academy of Sciences (No. Y60707WR04).

\section{References}

1 A. M. Omer, Water resources and freshwater ecosystems in Sudan, Renewable Sustainable Energy Rev., 2008, 12, 20662091.

2 M. Floerke, E. Kynast, et al., Domestic and industrial water uses of the past 60 years as a mirror of;socio-economic development: A global simulation study, NATO ASI Ser., Ser. I, 2013, 23, 144-156.

3 B. Tang and Z. Zhang, Essence of disposing the excess sludge and optimizing the operation of wastewater treatment: rheological behavior and microbial ecosystem, Chemosphere, 2014, 105, 1-13.

4 G. Asman and O. Şanl, Characteristics of Permeation and Separation for Acetic Acid-Water Mixtures Through Poly(Vinyl Alcohol) Membranes Modified with Poly(Acrylic Acid), Sep. Sci. Technol., 2003, 38, 1963-1980.

5 S. Simone, A. Figoli, S. Santoro, et al., Preparation and characterization of ECTFE solvent resistant membranes and their application in pervaporation of toluene/water mixtures, Sep. Sci. Technol., 2012, 90, 147-161.

6 Q. H. She, L. N. Chi, W. L. Zhou, et al., Overview of forward osmosis membrane separation technology: research and its application to water treatment, Environ. Sci. Technol., 2010, 33, 117-122.

7 R. Kiełczyński and M. Bryjak, Molecularly imprinted membranes for cinchona alkaloids separation, Sep. Sci. Technol., 2005, 41, 231-235.

8 J. Zhao, K. Zhang, D. Gao, et al., Optimization of $\mathrm{Ba}_{\mathrm{x}} \mathrm{Sr}_{1-}$ ${ }_{\mathrm{x}} \mathrm{Co}_{0.9} \mathrm{Nb}_{0.1} \mathrm{O}_{3}$-delta perovskite as oxygen semi-permeable membranes by compositional tailoring, Sep. Sci. Technol., 2010, 71, 152-159.

9 Y. N. Wang and C. Y. Tang, Protein fouling of nanofiltration, reverse osmosis, and ultrafiltration membranes-The role of hydrodynamic conditions, solution chemistry, and membrane properties, J. Membr. Sci., 2011, 376, 275-282.

10 S. Ramakrishna, Z. Ma, and T. Matsuura, Membrane and Membrane Separation Process, 2014.

11 T. Yuan, J. Meng, T. Hao, et al., A scalable method toward superhydrophilic and underwater superoleophobic PVDF membranes for effective oil/water emulsion separation, ACS Appl. Mater. Interfaces, 2015, 7, 14896-14904.

12 Y. Zhang, W. Yu, R. Li, et al., Novel conductive membranes breaking through the selectivity-permeability trade-off for Congo red removal, Sep. Purif. Technol., 2019, 211, 368-376.

13 A. C. Hinckley, C. Wang, R. Pfattner, et al., Investigation of a Solution-Processable, Nonspecific Surface Modifier for Low Cost, High Work Function Electrodes, ACS Appl. Mater. Interfaces, 2016, 8, 19658-19664.

14 Y. Chen, J. Teng, L. Shen, et al., Novel insights into membrane fouling caused by gel layer in a membrane bioreactor: Effects of hydrogen bonding, Bioresour. Technol., 2019, 276, 219-225.

15 J. Teng, M. Zhang, K. T. Leung, et al., A unified thermodynamic mechanism underlying fouling behaviors of soluble microbial products (SMPs) in a membrane bioreactor, Water Res., 2019, 149, 477-487.

16 M. Mondal and S. De, Preparation and characterization of PDMS-PVDF hydrophobic microporous membrane for membrane distillation, Desalination, 2015, 370, 63-71.

17 W. Yu, Y. Liu, Y. Xu, et al., A conductive PVDF-Ni membrane with superior rejection, permeance and antifouling ability via electric assisted in situ aeration for dye separation, $J$. Membr. Sci., 2019, 581, 401-412.

$18 \mathrm{~J}$. Li, J. Miao, X. Shao, Y. Xu, et al., Surface modification of PVDF porous membranes, Chin. J. Polym. Sci., 2013, 31, 994-1001.

19 X. Huang, W. Wang, Y. Liu, et al., Treatment of oily waste water by PVP grafted PVDF ultrafiltration membranes, Chem. Eng. J., 2015, 273, 421-429.

20 S. Gong, H. Jeon, H. Lee, et al., Effects of an Integrated Separator/Electrode Assembly on Enhanced Thermal Stability and Rate Capability of Lithium-Ion Batteries, ACS Appl. Mater. Interfaces, 2017, 9, 17814-17821.

21 L. Shen, Y. Zhang, W. Yu, et al., Fabrication of hydrophilic and antibacterial poly(vinylidene fluoride) based separation membranes by a novel strategy combining radiation grafting of poly(acrylic acid) (PAA) and electroless nickel plating, J. Colloid Interface Sci., 2019, 543, 64-75. 
22 Y. Zhao, W. Yu, R. Li, et al., Electric field endowing the conductive polyvinylidene fluoride (PVDF)-graphene oxide (GO)-nickel (Ni) membrane with high-efficient performance for dye wastewater treatment, Appl. Surf. Sci., 2019, 483, 1006-1016.

23 S. Wongchitphimon, R. Wang, R. Jiraratananon, et al., Effect of polyethylene glycol (PEG) as an additive on the fabrication of polyvinylidene fluoride-co-hexafluropropylene (PVDFHFP) asymmetric microporous hollow fiber membranes, $J$. Membr. Sci., 2011, 369, 329-338.

24 D. Pozzi, V. Colapicchioni, G. Caracciolo, et al., Effect of polyethyleneglycol (PEG) chain length on the bio-nanointeractions between PEGylated lipid nanoparticles and biological fluids: from nanostructure to uptake in cancer cells, Nanoscale, 2014, 6, 2782-2792.

25 N. S. Kacem, F. Delporte, Y. Muhovski, et al., In vitro screening of durum wheat against water-stress mediated through polyethylene glycol, Genet. Eng. Biotechnol. J., 2017, 15, 237-247.

26 K. Thuresson, F. E. Antunes, M. G. Miguel, et al., The association between a non-ionic microemulsion and hydrophobically modified PEG. A rheological investigation, Prog. Colloid Polym. Sci., 2004, 123, 40-43.

27 J. Zhang, Z. Xu, W. Mai, et al., Improved hydrophilicity, permeability, antifouling and mechanical performance of PVDF composite ultrafiltration membranes tailored by oxidized low-dimensional carbon nanomaterials, J. Mater. Chem. A, 2013, 1, 3101-3111.

28 M. Yang, L. Wang, T. Hou, et al., Controlling of the electronic properties of WS2 and graphene oxide heterostructures from first-principles calculations, J. Mater. Chem. C, 2016, 5, 201207.

29 A. Falin, Q. Cai, S. Ejg, et al., Mechanical properties of atomically thin boron nitride and the role of interlayer interactions, Nat. Commun., 2017, 8, 15815-15823.

30 Z. Wang and B. Mi, Environmental Applications of 2D Molybdenum Disulfide $\left(\mathrm{MoS}_{2}\right)$ Nanosheets, Environ. Sci. Technol., 2017, 51, 8229-8244.

$31 \mathrm{X}$. Song, J. $\mathrm{Hu}$ and $\mathrm{H}$. Zeng, Two-dimensional semiconductors: recent progress and future perspectives, $J$. Mater. Chem. C, 2013, 1, 2952-2969.

32 J. Zhu, J. Wang, J. Hou, et al., Graphene-based antimicrobial polymeric membranes: a review, J. Mater. Chem. A, 2017, 5, 6676-6793.

33 Y. He, R. Hu, Y. Zhong, et al., Graphene oxide as a water transporter promoting germination of plants in soil, Nano Res., 2018, 11, 1928-1937.

34 W. Yu, Y. Liu, Y. Xu, R. Li, J. Chen, B.-Q. Liao, L. Shen and H. Lin, J. Membr. Sci., 2019, 581, 401-412.

35 Z. Wang, H. Yu, J. Xia, et al., Novel GO-blended PVDF ultrafiltration membranes, Desalination, 2012, 299, 50-54.
36 Z. J. Jiang, Z. Jiang, X. Tian, et al., Sulfonated Holey Graphene Oxide (SHGO) Filled Sulfonated Poly(ether ether ketone) Membrane: The Role of Holes in the SHGO in Improving Its Performance as Proton Exchange Membrane for Direct Methanol Fuel Cells, ACS Appl. Mater. Interfaces, 2017, 9, 20046-20056.

37 Y. Hu, Y. Wang, Z. Zeng, et al., PEGlated Graphene as Nanoadditive for Enhancing the Tribological Properties of Water-based Lubricant, Carbon, 2018, 137, 41-48.

38 S. Zalipsky, Functionalized poly(ethylene glycol) for preparation of biologically relevant conjugates, ChemInform, 1995, 26, 150-165.

39 D. Y. Zuo, B. K. Zhu, J. H. Cao, et al., Influence of AlcoholBased Nonsolvents on the Formation and Morphology of PVDF Membranes in Phase Inversion Process, Chin. J. Polym. Sci., 2006, 24, 281-289.

40 N. Meng, R. C. E. Priestley, Y. Zhang, et al., The effect of reduction degree of GO nanosheets on microstructure and performance of PVDF/GO hybrid membranes, J. Membr. Sci., 2016, 501, 169-178.

41 M. Na, R. C. E. Priestley, Y. Zhang, et al., The effect of reduction degree of GO nanosheets on microstructure and performance of PVDF/GO hybrid membranes, J. Membr. Sci., 2016, 501, 169-178.

42 V. Vatanpour, S. S. Madaeni and L. Rajabi, Boehmite nanoparticles as a new nanofiller for preparation of antifouling mixed matrix membranes, J. Membr. Sci., 2012, 401-402, 132-143.

43 L. Zhu, F. Liu, X. Yu, et al., Poly(Lactic Acid) Hemodialysis Membranes with Poly(Lactic Acid)-block-Poly(2Hydroxyethyl Methacrylate) Copolymer As Additive: Preparation, Characterization, and Performance, ACS Appl. Mater. Interfaces, 2015, 7, 17748-17755.

44 Z. Xu, J. Zhang, M. Shan, et al., Organosilane-functionalized graphene oxide for enhanced antifouling and mechanical properties of polyvinylidene fluoride ultrafiltration membranes, J. Membr. Sci., 2014, 458, 1-13.

45 L. Yan, Y. S. Li, C. B. Xiang, et al., Effect of nano-sized $\mathrm{Al}_{2} \mathrm{O}_{3}$ -particle addition on PVDF ultrafiltration membrane performance, J. Membr. Sci., 2006, 276, 162-167.

46 C. Zhao, X. Xu, C. Jie, et al., Effect of graphene oxide concentration on the morphologies and antifouling properties of PVDF ultrafiltration membranes, J. Environ. Chem. Eng., 2013, 1, 349-354.

47 S. Ayyaru and Y. H. Ahn, Application of sulfonic acid group functionalized graphene oxide to improve hydrophilicity, permeability, and antifouling of PVDF nanocomposite ultrafiltration membranes, J. Membr. Sci., 2017, 525, 210219. 